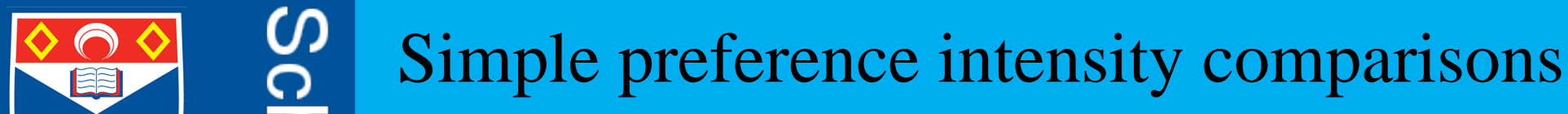

University of

Georgios Gerasimou

St Andrews

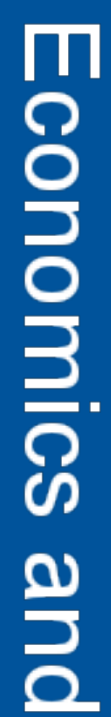

School of Economics and Finance Discussion Paper No. 1905 1 Jun 2019 (revised 3 Feb 2022)

JEL Classification: JEL: B21, C88, D11, D63, D70, D90

Keywords: Ordinal preference intensities; Preference intensity functions; Revealed preference intensity 


\title{
Simple preference intensity comparisons
}

\author{
Georgios Gerasimou ${ }^{1}$ \\ School of Economics $\mathcal{E}$ Finance, University of St Andrews, Scotland, United Kingdom \\ Dedicated to Paraskevi Kalliga-Gerasimou
}

\begin{abstract}
We propose and analyse a general model of simple preference intensity comparisons. The model encompasses those that belong to the utility-difference class, has transparent behavioural underpinnings and features purely ordinal uniqueness properties. Its empirical content is characterized by an easily testable condition on dual behavioural data that include choices and additional observables with intensity-revealing potential that are often elicited in experimental/empirical work, such as survey ratings, response times or willingness to pay.
\end{abstract}

Keywords: Ordinal preference intensities, Preference intensity functions, Revealed preference intensity

\section{Introduction}

The idea that decision makers not only have preferences over the choice alternatives of interest but may also be capable of ranking differences in preference intensities between those alternatives has been present -often implicitlyin many areas of economics, including decision theory and experiments, welfare and public economics, political economy, voting, matching and market design. All existing modelling approaches in this large and diverse body of work assume that an individual's preference intensities are ultimately expressible on a cardinal or pseudo-cardinal utility scale. This paper shows instead how it is possible to model the decision maker as able to make simple and purely ordinal preference intensity comparisons such as "I prefer my child enrolling in School A than in School B more than I prefer it enrolling in School C than in School D" without assuming that such comparisons can also be quantified in any way.

Our approach takes Paul Samuelson's (1938) bivariate formulation of neoclassical cardinal utility representations as a starting point and extends it in a natural and intuitive but hitherto unexplored direction. Specifically, we propose the general class of preference intensity functions that mimic ordinal utility ones by associating each pair of alternatives with a numerical value in a way that preserves the intensity ordering. In particular, letting $s$ be such a function, $s(a, b) \geq s(c, d)$ holds if and only if $a$ is preferred to $b$ at least as much as $c$ is to $d$. The key novelty of such preference intensity representations that formally distinguishes them from utility-difference ones (or bivariate representations that are formally equivalent to those) is a property that we refer to as lateral consistency. This requires that whenever $a$ is preferred to $b$ and $b$ to $c$, then $a$ is preferred to $c$ no less than $a$ is to $b$ or $b$ to $c$, i.e. $s(a, c) \geq \max \{s(a, b), s(b, c)\}$. The condition ensures conceptual harmony between and within the preference and preference intensity relations, and also establishes transitivity of the former without forcing -as is done by utility-difference models- that the value capturing the intensity difference between $a$ and $c$ be the sum of the values of the intensity differences between $a$ and $b$ and between $b$ and $c$. By not imposing this additivity requirement, the model is characterized by the standard Weak Order and Reversal axioms together with an intuitive Translocation Consistency one.

\footnotetext{
${ }^{1}$ A previous version of this paper was circulated in December 2017 as "Preference intensity representation and revelation". Thanks are due to conference and seminar audiences at NASMES 2018 (Davis), SARP 2018 (Oxford), RUD 2019 (Paris), EEA/ESEM 2019 (Manchester), St Andrews, Royal Holloway, Warwick and especially to Jean Baccelli, Tore Ellingsen, John Quah, Peter Wakker and the anonymous referees and associate editor for very helpful comments. Any errors are the author's own.

Email address: gg26@st-andrews . ac.uk (Georgios Gerasimou)
} 
Then, generalizing Echenique and Saito (2017) who motivated their analysis based on choice and response-time data, we also show that the testable Congruent Monotonicity axiom that we introduce characterizes preferenceintensity rationalizable datasets that comprise finitely many binary choices and an additional menu-specific observable or foregone resource with intensity-revealing potential such as survey-based intensity ratings, response times or willingness to pay. When rationalizable in this way, the relevant dataset can be thought of as being generated by a decision maker whose preferences and intensity comparisons are representable by a preference intensity function that is strictly monotonic in the observed resource. By using preference intensity functions instead of difference-preserving utility functions as its building block, this more general notion of rationalizability can account for substantially more behaviour without sacrificing completeness, transitivity or conceptual harmony between the revealed preference and intensity relations.

The next section introduces preference intensity relations and the relevant notation. The following section defines the benchmark class of utility-difference representations for such relations and summarizes some important conceptual and analytical challenges that are associated with existing manifestations of this modelling approach. Motivated by this discussion, Section 4 introduces and axiomatically characterizes preference intensity functions, clarifying why such representations are genuinely ordinally unique and how they include utility-difference representations as special cases. Section 5 presents the revealed preference intensity analysis and the last section concludes. All proofs appear in the Appendix.

\section{Preliminaries}

Assumed throughout is a set $X$ of general choice alternatives and a primitive binary relation $\succsim$ on $X \times X$ that will be thought of as a preference intensity relation on $X$. Such $\succsim$ can either be viewed as a binary relation on $X \times X$ or as a quaternary relation on $X .^{3}$ The comparison $(a, b) \succsim(c, d)$ will be interpreted as suggesting that $a$ is weakly preferred to $b$ at least as much as $c$ is to $d$; that $a$ is weakly inferior to $b$ at most as much as $c$ is to $d$; etc. Although we will return to this issue in more detail below, it is worth stating from the outset that the comparison $(a, b) \succsim(c, d)$ admits several possible data-driven definitions, of which the following are some examples:

1. Questionnaires/surveys: $a$ is stated to be preferred to $b$ more than $c$ is to $d$.

2. Willingness to pay: higher for $a$ at menu $\{a, b\}$ than for $c$ at $\{c, d\}$.

3. Choice probabilities: $a$ is chosen more frequently at $\{a, b\}$ than $c$ is at $\{c, d\}$.

4. Response times: $a$ is chosen faster at $\{a, b\}$ than $c$ is at $\{c, d\}$.

5. Physical distance/effort: more is travelled/exerted in choosing $a$ from $\{a, b\}$ than $c$ from $\{c, d\}$.

6. Biological indicators: e.g. higher neural activity is observed when choosing $a$ at $\{a, b\}$ than $c$ at $\{c, d\}$.

We will say that a preference intensity relation $\succsim$ induces an ordinary preference relation $\succsim$ on $X$ when the latter is defined by

$$
\begin{aligned}
& a \succsim b \quad(a, b) \succsim(b, a) \\
& \Longleftrightarrow \quad(a, b) \succsim(c, c) \text { for any } c \in X .
\end{aligned}
$$

(The equivalence between (1) and (2) will hold in our environment.) Intuitively, if the intensity difference between $a$ and $b$ is ranked above the "zero" intensity difference between any alternative $c$ and itself, then a "positive" such difference exists within the pair $(a, b)$, which can be interpreted as a preference for $a$ over $b$. Similarly, if a transition from $b$ to $a$ is preferred to one from $a$ to $b$, then the logical conclusion is that $a$ is preferred to $b$. The asymmetric and symmetric parts of relations $\grave{\succsim}$ and $\succsim$ will be denoted, respectively, $\dot{>}, \dot{\sim}$ and $>, \sim$.

\section{The utility-difference approach and its limitations}

The problem of representing a complete and transitive preference intensity relation by a numerical function has been approached in the literature invariably through the class of utility-difference models. We state below the formal definition of these representations and also distinguish between cardinal and pseudo-cardinal ones.

${ }^{3}$ The first economist to formally study preference intensities using quaternary relations appears to be Ragnar Frisch (1926, 1971). 
Definition 1. A binary relation $\grave{\succsim}$ on a set $X \times X$ admits a utility-difference representation if there exists a function $u: X \rightarrow \mathbb{R}$ such that, for all $a, b, c, d \in X$,

$$
(a, b) \succsim(c, d) \Longleftrightarrow u(a)-u(b) \geq u(c)-u(d) .
$$

When such $u$ exists and is unique up to a positive affine transformation, then (3) is a cardinal utility representation of $\dot{\succsim}$. If, instead, such $u$ exists but does not have this uniqueness property, then (3) is a pseudo-cardinal utility representation of $\succsim$. We present below some of the critical insights that have been offered by the extended study of these representations over the past several decades that are particularly relevant for this paper's motivation and focus. ${ }^{4}$

\section{Riskless cardinal utility}

This model postulates the existence of a cardinal utility function $u$ on a general choice domain. It implies that not only are the agent's intensity comparisons well-defined and consistent, but they are also precise to a behaviourally questionable degree. Specifically, if $\dot{\succsim}$ is represented by such $u$, then the statement $(a, b) \succsim(c, d)$ is equivalent to a utility-difference ratio $\frac{u(a)-u(b)}{u(c)-u(d)}:=r$ that is invariant with respect to all permissible transformations of $u$. Assuming, for simplicity, that both utility differences are positive, this can be read as " $a$ is preferred to $b$ exactly $r$ times as much as $c$ is preferred to $d$ ". In addition, Basu (1982) showed that when such a cardinal $u$ exists and $u(X)$ is an interval, then utility differences of any finite order are ranked, not just the first-order differences in (3). Finally, a technical solvability condition (Alt, 1936, 1971; Suppes and Winet, 1955; Shapley, 1975; Köbberling, 2006) must be satisfied by the intensity relation for this model to be well-defined. This condition requires the domain to be infinite in order to prevent trivialities in the intensity relation (see Krantz et al., 1971; Theorem 5, p. 168).

\section{von Neumann-Morgenstern cardinal utility}

The cardinally unique utility differences between pairs of riskless alternatives that can be obtained by lottery comparisons under the von Neumann and Morgenstern (1947) expected-utility model are often interpreted as indicating differences in preference intensities between those alternatives. It is well-known, however, that such utility differences confound the individuals' preference intensities over riskless alternatives with her attitudes toward risk (Arrow, 1951; Luce and Raiffa, 1957; Schoemaker, 1982; Hammond, 1991; Ellingsen, 1994; Abdellaoui et al., 2007; Baccelli and Mongin, 2016).

\section{Cardinal utility from stochastic choices}

Debreu's (1958) axiomatization of cardinal utility is based on comparisons of binary choice probabilities and, like the riskless model above, also requires the choice set to be infinite. As pointed out in Davidson and Marschak (1959), moreover, this model effectively rules out choice probabilities that are either 0 or 1 , for otherwise one could end up with counter-intuitive non-monotonicities such as $u(\$ 5)=u(\$ 5,000)$. This difficulty is circumvented by the Positivity axiom which requires all choice probabilities to be strictly positive. Based on an argument in Baccelli and Mongin (2016), however, one might then respond that such genuine choice stochasticity precludes the possibility of the decision maker behaving like an ordinal utility maximizer, yet the model portrays her as one whose behaviour is describable by maximization of a utility function that has even more refined uniqueness properties.

\section{Riskless pseudo-cardinal utility from ordered preferences}

This model postulates the existence of some $u$ in the sense of (3) without also requiring its cardinal uniqueness, and is applicable on finite domains in non-trivial ways. Yet, its behavioural implications are unclear due to a testable but impossible to fully interpret Cancellation axiom that lies at the heart of its axiomatization (Scott, 1964; Adams, 1965; Luce and Suppes, 1965; Tversky, 1967). In addition, the uniqueness property of $u$ in this model is a complicated and relation-specific entity that lies between ordinal and cardinal and no longer allows $u$ to act as a unit of measurement (see Theorem 2, p. 431 in Krantz et al., 1971). ${ }^{5}$

\footnotetext{
${ }^{4}$ For detailed and complementary accounts of these models, the debates they have been associated with and their implications for economic analysis, the reader is also referred to Luce et al. (1990), Krantz et al. (1971), Suppes et al. (1989), Hammond (1991), Ellingsen (1994), Mandler (1999), Falmagne (2002), Bossert and Weymark (2004), Fleurbaey and Hammond (2004), Abdellaoui et al. (2007), Baccelli and Mongin (2016).

${ }^{5}$ On a related note, the permissible transformations of a utility function over wealth that preserve the property of diminishing marginal utility also lie between these two extreme polar cases and are studied in Mandler (2006).
} 


\section{Riskless pseudo-cardinal utility from semi-ordered preferences}

An alternative to rational utility-difference representations of preference intensity relations takes as primitive a semi-order $P$ on $X$ that is representable by a pair $(u, \delta)$ where $u: X \rightarrow \mathbb{R}$ and $\delta>0$ satisfy $a P b \Leftrightarrow u(a)-u(b)>\delta$ (Luce, 1956). A semi-order is a special case of a strict partial order whose incomparability relation is often interpreted as an intransitive indifference relation. The uniqueness properties of this model are also between ordinal and cardinal (Roberts, 1979), and value differences such as $u(a)-u(b)>n \cdot \delta>u(c)-u(d)>\delta$ are sometimes taken to suggest that $a$ is preferred to $b$ at least $n$ times more than $c$ is to $d$. In the special case where $P$ is total/complete so that $\delta=0$, this pseudo-cardinal uniqueness property of $u$ that motivates its preference-intensity interpretation disappears.

\section{Preference intensity functions}

\subsection{General properties and characterization in finite domains}

From now on the analysis will revolve around the following concept.

Definition 2. A binary relation $\dot{\succsim}$ on a set $X \times X$ is representable by a preference intensity function if there exists a mapping $s: X \times X \rightarrow \mathbb{R}$ such that, for all a, $b, c, d \in X$,

$$
\begin{aligned}
(a, b) \succsim(c, d) & \Longleftrightarrow s(a, b) \geq s(c, d) \\
s(a, b) & =-s(b, a) \\
s(a, b) \geq 0, s(b, c) \geq 0 & \Longrightarrow s(a, c) \geq \max \{s(a, b), s(b, c)\},
\end{aligned}
$$

where $s$ is unique up to an odd and strictly increasing transformation ${ }^{6}$ in the sense that $t: X \times X \rightarrow \mathbb{R}$ also represents $\succsim$ as in (4) if and only if $t=f \circ$ s for some $f: \mathbb{R} \rightarrow \mathbb{R}$ that is odd and strictly increasing in $s(X \times X)$.

As captured by the order-preservation requirement (4a), a preference intensity function represents a binary relation on the set of pairs of alternatives in exactly the same way that an ordinal utility function represents a binary relation on the set of alternatives. This analogy also motivates its bivariate nature. Under (4a), the skew-symmetry condition (4b) means that one need only look at the sign of $s(a, b)$ to infer whether the agent strictly prefers $a$ to $b$ (positive sign), if the opposite is true (negative sign), or if she is indifferent between $a$ and $b$ (zero value). Therefore, a preference intensity function that represents such a relation also represents the ordinary preferences induced by it in the sense of (1). Moreover, in the special case where the intensity relation also admits a utility-difference representation by means of a function $u$, skew-symmetry allows $s$ to be defined by $s(a, b):=u(a)-u(b)$. Apart from these implications of the particularly convenient normalization that is afforded by skew-symmetry, it will be formally established below that this condition is otherwise void of behavioural content.

The last defining property of preference intensity functions, (4c), will be referred to as lateral consistency. Under the maintained assumption that (4a) and (4b) are in place, it is interpretable as requiring that if $a$ is weakly preferred to $b$ and $b$ to $c$, then $a$ is weakly preferred to $c$ at least as much as $a$ is to $b$ and $b$ is to $c$. Lateral consistency therefore imposes the intuitive restriction that the preference and preference-intensity comparisons are in conceptual harmony in the sense that as the decision maker goes down her preference ranking from $a$ to $b$ and from $b$ to $c$, her preference intensity between the "remote" alternatives $a$ and $c$ in this ranking is higher than that between the "proximal" alternatives $a, b$ and $b, c$. In addition, (4c) ensures that the preferences induced by the preference intensity relation represented by $s$ are transitive, which is not implied by (4a) and (4b) alone.

Finally, preference intensity functions are essentially ordinally unique, with strictly increasing transformations also required to be odd only in order to preserve the normalization offered by skew-symmetry. Towards establishing the behavioural irrelevance of skew-symmetry and odd transformations in this model, we introduce next the following seemingly more general class of functions where both these properties are relaxed and lateral consistency is slightly modified, and later show that both are characterized by the same axioms.

\footnotetext{
${ }^{6}$ A function $f: \mathbb{R} \rightarrow \mathbb{R}$ is odd in $A \subseteq \mathbb{R}$ if $f(-z)=-f(z)$ holds for all $z \in A$.
} 
Definition 3. A binary relation $\succsim$ on a set $X \times X$ is representable by a general preference intensity function if there exists a mapping $g: X \times X \rightarrow \mathbb{R}$, unique up to a strictly increasing transformation, such that, for all a, $b, c, d \in X$,

$$
\begin{aligned}
(a, b) \succsim(c, d) & \Longleftrightarrow g(a, b) \geq g(c, d) \\
g(a, b) \geq g(c, d) & \Longrightarrow g(d, c) \geq g(b, a) \\
\min \{g(a, b), g(b, c)\} \geq g(d, d) & \Longrightarrow g(a, c) \geq \max \{g(a, b), g(b, c)\} .
\end{aligned}
$$

For a function $g$ that satisfies (5a)-(5c) but not (4a)-(4c), skew-symmetry is replaced by a weaker condition that retains the same ordering restriction without imposing a sign requirement on the function's values. Thus, $a \succsim b \Leftrightarrow s(a, b) \geq 0$ in (4b) becomes $a \succsim b \Leftrightarrow s(a, b) \geq s(b, a)$ in (5b). Consistent with this observation, the comparison of (4c) and (5c) suggests that the real "zero" in a general preference intensity function is its value at any point on the diagonal of $X \times X$ where, by definition, there is "zero" preference intensity difference in moving from the second alternative within the pair to itself. Finally, without a need for skew-symmetry to be accounted for in intensity-preserving transformations of a function satisfying (5a)-(5c), the uniqueness property in the class of general preference intensity functions coincides with that of standard ordinal transformations.

We now turn to the axioms that will be imposed on the relation $\succsim$.

\section{Weak Order}

For all $a, b, c, d \in X,(a, b) \succsim(c, d)$ or $(c, d) \succsim(a, b)$.

For all $a, b, c, d, e, f \in X,(a, b) \succsim(c, d) \succsim(e, f)$ implies $(a, b) \succsim(e, f)$.

Weak Order imposes completeness and transitivity on the relation $\dot{\succsim}$. Hence, it requires the decision maker to be able to make preference comparisons universally and consistently.

\section{Reversal}

For all $a, b, c, d \in X,(a, b) \succsim(c, d)$ implies $(d, c) \succsim(b, a)$.

Reversal too is a standard condition and allows for the relation $\succsim$ to be interpreted as a preference intensity relation by requiring that whenever the transition from $b$ to $a$ is more desirable than that from $d$ to $c$, then the transition from $c$ to $d$ also be preferable to that from $a$ to $b$. If $a$ is preferred to $b$ more than $c$ is to $d$, for example, then since the intensity difference between $c$ and $d$ is ranked below the one between $a$ and $b$, the transition from $c$ to $d$ should be associated with a lower psychological cost than that from $a$ to $b$, and therefore, intuitively, $(d, c) \dot{\succsim}(b, a)$ should hold.

\section{Translocation Consistency}

For all $a, b, c \in X,(a, c) \succsim(b, c)$ implies $(a, b) \succsim(b, a)$.

Recalling also the definition of induced preferences in (1), this condition requires that whenever the agent prefers the transition from $c$ to $a$ more than the one from $c$ to $b$, then she prefers $a$ to $b$. As will be shown below, under Weak Order and Reversal, Translocation Consistency turns out to be equivalent to the familiar

\section{Separability}

For all $a, b, c, d \in X,(a, c) \succsim(b, c)$ implies $(a, d) \succsim(b, d)$.

We note that, alongside Weak Order, Reversal and several additional axioms, a stronger version of Separability appears in the first axiomatization of the riskless cardinal utility model that was given in Alt $(1936,1971)$.

Theorem 1. The following are equivalent for a binary relation $\succsim$ on a finite set $X \times X$ :

1. $\succsim$ satisfies Weak Order, Reversal and Translocation Consistency.

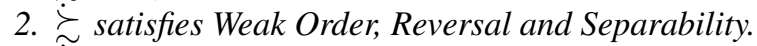

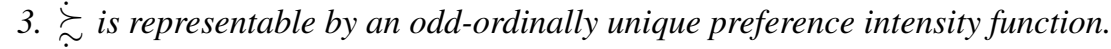

4. $\succsim$ is representable by an ordinally unique general preference intensity function. 
The last equivalence in Theorem 1 clarifies that the convenient normalization offered by skew-symmetry is without loss of generality. In addition, whenever such an assumption is useful in some application of the model, it is also without loss to assume a canonical such representation where, as in Example 1 below, the function $s$ is onto a range of consecutive integers that are symmetric around zero. The proof, in fact, proceeds by constructing such a function.

Example 1. Let $X:=\{a, b, c, d\}$ and consider the intensity relation $\dot{\succsim}$ on $X$ defined by

$$
(a, d) \quad \dot{>}(b, d) \quad \dot{>}(a, c) \quad \dot{>}(b, c) \quad \dot{>}(a, b) \quad \dot{>}(c, d) .
$$

As per the Reversal axiom, assume further that $\left(a^{\prime}, b^{\prime}\right) \succsim\left(c^{\prime}, d^{\prime}\right)$ implies $\left(d^{\prime}, c^{\prime}\right) \succsim\left(b^{\prime}, a^{\prime}\right)$ for $a^{\prime}, b^{\prime}, c^{\prime}, d^{\prime} \in X$. This means that the pair $(c, d)$ is followed by all pairs $(z, z)$ that lie on the diagonal of $X \times X$, which are then followed by the pair $(d, c)$, etc. Thus extended, $\succsim$ satisfies Weak Order, Reversal and Translocation Consistency/Separability. Now suppose to the contrary that there is $u: X \rightarrow \mathbb{R}$ that represents $\dot{\succsim}$ as in (3). It follows from $(a, b) \dot{\succ}(c, d)$ and $(b, d) \dot{>}(a, c)$ that

$$
\begin{aligned}
& u(a)-u(b)>u(c)-u(d) \\
& u(a)-u(c)<u(b)-u(d)
\end{aligned}
$$

and subtracting the second inequality from the first results in the contradiction $u(c)-u(b)>u(c)-u(b)$. Note that this relation is not utility-difference representable even though, in addition to the above axioms, it also satisfies what is often referred to as the Concatenation axiom -originally introduced and criticized in Samuelson (1938)- whereby $(a, b) \dot{\succsim}\left(a^{\prime}, b^{\prime}\right)$ and $(b, c) \dot{\succsim}\left(b^{\prime}, c^{\prime}\right)$ implies $(a, c) \dot{\succsim}\left(a^{\prime}, c^{\prime}\right)$. Note, finally, that $\dot{\succsim}$ is representable by a canonical preference intensity function that sets $s(a, d):=6, s(b, d):=5, s(a, c):=4, s(b, c):=3, s(a, b):=2, s(c, d):=1$ and $s\left(a^{\prime}, b^{\prime}\right):=-s\left(b^{\prime}, a^{\prime}\right)$.

\subsection{Infinite domains}

We now turn to the case where $X$ is an infinite set by endowing it with the structure of a connected and separable metric space. This implies that finite products of that space -once endowed with the topologies induced by their respective product metrics- are also connected and separable metric spaces. The following continuity axiom on such domains is standard and, in the special case where the primitive is a preference intensity relation on $X=\mathbb{R}_{+}$, it appears, for example, in Shapley (1975).

\section{Continuity}

For all $a, b \in X$, the sets $\mathcal{L}_{\succsim}(a, b):=\{(c, d) \in X \times X:(a, b) \succsim(c, d)\}$ and $\mathcal{U}_{\succsim}(a, b):=\{(c, d) \in X \times X:(c, d) \succsim(a, b)\}$ are closed.

Theorem 2. The following are equivalent for a binary relation $\succsim$ on a connected and separable metric space $X \times X$ : 1. $\succsim$ satisfies Weak Order, Reversal, Translocation Consistency and Continuity.

2. $\succsim$ is representable by a continuous general preference intensity function.

Example 2. Let $X:=\mathbb{R}_{++}^{2}$ and define the preference intensity functions $s_{1}, s_{2}: X \times X \rightarrow \mathbb{R}$ by

$$
\begin{aligned}
& s_{1}(a, b):=a_{1} a_{2}-b_{1} b_{2}, \\
& s_{2}(a, b):=\frac{a_{1}}{b_{1}}-\frac{b_{1}}{a_{1}}+\frac{a_{2}}{b_{2}}-\frac{b_{2}}{a_{2}} .
\end{aligned}
$$

The first function is obviously utility-difference decomposable, with $s_{1}(a, b) \equiv u(a)-u(b)$ for $u(x):=x_{1} x_{2}$. The second, however, is not. Indeed, both super- and sub-additivities $s_{2}(a, c)>s_{2}(a, b)+s_{2}(b, c)$ and $s_{2}(a, c)<s_{2}(a, b)+$ $s_{2}(b, c)$ occur in general, in contrast to the additivity condition that is necessary and sufficient for such a decomposition (see equation (7) and the related discussion in Section 4.3 below). Finally, Figure 1 shows that the preferences $\succsim_{1}$ and $\succsim_{2}$ induced by the intensities $\dot{\succsim}_{1}$ and $\dot{\succsim}_{2}$ that are represented by $s_{1}$ and $s_{2}$ are generally distinct, which in turn implies that $\dot{\succsim}_{1}$ and $\dot{\succsim}_{2}$ are themselves distinct. 
Figure 1: The preferences induced by $s_{1}$ (dashed indifference curve) are generally distinct from those induced by $s_{2}$ (thick).

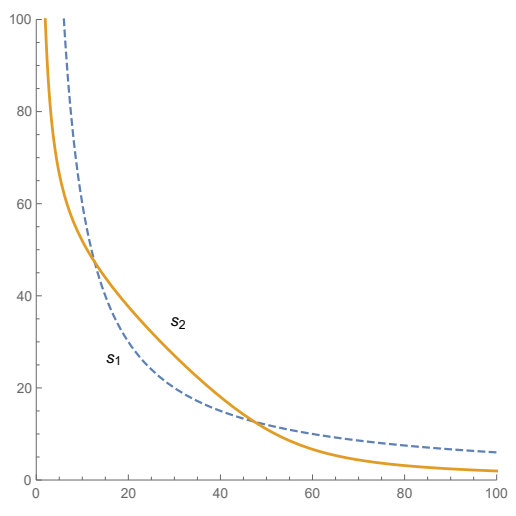

\subsection{Special cases}

The concept introduced next will be helpful in clarifying the formal relationship between preference-intensity and utility-difference representations.

Definition 4. A binary relation $\succsim$ on a set $X \times X$ is representable by an additive preference intensity function $s: X \times X \rightarrow \mathbb{R}$ if, for all $a, b, c \in X$,

$$
s(a, c)=s(a, b)+s(b, c)
$$

Additivity is not satisfied by preference intensity functions in general, and both sub- and super-additive deviations generally occur within the context of the same such representation (cf Examples 1 and 2). When this condition is satisfied, however, it implies both (4b) and (4c). Additive preference intensity functions are therefore characterized by (4a) and (7) only.

An intensity relation $\succsim$ is representable by an additive preference intensity function if and only if it admits a (pseudo-)cardinal utility representation as in (3). The equivalence -which also holds in infinite domains- follows from Sincov's functional equation ${ }^{7}$ that is defined by (7) and the solution to that equation, a unique function $u: X \rightarrow \mathbb{R}$ such that $s(a, b) \equiv u(a)-u(b)$. This equivalence shows that the proposed model nests the utility-difference model whenever the underlying preference intensity order is representable by a preference intensity function $s$ that takes this particular additively separable form. Although the equivalence was noted by Samuelson (1938) in his bivariate reformulation and criticism of cardinal utility representations, we emphasize that Samuelson did not suggest to use the bivariate approach as a potentially more general way to represent preference intensity relations. In particular, despite his critical approach towards what we are referring to as additive preference intensity functions, Samuelson did not propose a way of relaxing this property in order to alleviate the concerns that he and some of his contemporaries (e.g. Phelps-Brown, 1934) had raised about the cardinal utility model, or, from a more modern perspective, the utilitydifference approach more generally. This paper appears to be the first that takes this stand and relaxes additivity with the far less demanding but still non-trivially structured lateral consistency condition.

Lateral consistency in turn bears a structural similarity with -but is logically and conceptually distinct from- what is known in the random choice literature as Strong Stochastic Transitivity (SST). Before stating this condition, let us recall that, given the collection $\mathcal{B}_{X}$ of all binary menus on a set $X$, a binary random choice model on $X$ is a function $\rho: X \times \mathcal{B}_{X} \rightarrow[0,1]$ such that, for all $a, b \in X, \rho(a,\{a, b\})+\rho(b,\{a, b\})=1$. Such a model $\rho$ is said to satisfy SST if $\rho(a,\{a, b\}) \geq \frac{1}{2}$ and $\rho(b,\{b, c\}) \geq \frac{1}{2}$ implies $\rho(a,\{a, c\}) \geq \max \{\rho(a,\{a, b\}), \rho(b,\{b, c\})\}$ (we will say that it satisfies $\mathrm{SST}^{*}$ if the last inequality is strict whenever one of the first two is also strict). Therefore, interpreting $\rho(a,\{a, b\}) \geq \frac{1}{2}$ as suggesting that the decision maker - whether a single person or, as is sometimes the case in practice, the average in a sample-probably prefers $a$ to $b$, SST requires that $a$ be probably preferred to $c$ more than $a$ is to $b$ and $b$ is to $c$.

\footnotetext{
${ }^{7}$ See, for example, Theorem 2, p. 356 in Aczél (1966) or pp. 97-98 in Falmagne (2002).
} 
Following Debreu (1958), one might also think of the comparison $\rho(a,\{a, b\}) \geq \rho(c,\{c, d\})$ as suggesting that $a$ is probably preferred to $b$ more than $c$ is to $d$. More formally, we can define the random preference intensity relation $\dot{~}$ that is induced by a binary random choice model $\rho$ on $X$ by

$$
(a, b) \succsim(c, d) \Longleftrightarrow \rho(a,\{a, b\}) \geq \rho(c,\{c, d\}) .
$$

As was also noted in Section 2 , such $\succsim$ is a special case of a preference intensity relation on the set $X$ that is simply defined by the algebraic differences between the particular real numbers that correspond to choice probabilities. Debreu (1958) imposed sufficient structure on the -necessarily infinite- set $X$ and on the binary random choice model $\rho$ for the relation $\succsim$ induced by it to admit a non-trivial cardinal utility difference representation in the sense of (3). The more general class of representations of binary random choice models where the utility function is not necessarily cardinally unique and $X$ can be finite without any trivialities arising is the class of Fechnerian representations (see Falmagne (2002) and references therein). These postulate the existence of some $u: X \rightarrow \mathbb{R}$ and a strictly increasing $F: \mathbb{R} \rightarrow \mathbb{R}$ such that

$$
\rho(a,\{a, b\})=F(u(a)-u(b))
$$

and may be thought of as corresponding to the strictly-stochastic special case of the general model of non-cardinal utility differences that was discussed earlier. A more general class of binary random choice models are known as simply scalable and postulate instead the existence of some $u: X \rightarrow \mathbb{R}$ and a function $F: \mathbb{R}^{2} \rightarrow \mathbb{R}$ that is strictly increasing (decreasing) in its first (second) argument and satisfies

$$
\rho(a,\{a, b\})=F(u(a), u(b)) .
$$

The monotonicity properties of this $F$, together with the fact that the pair $(u, F)$ is no longer required to preserve utility differences as in (8), immediately establishes the greater generality of simply scalable relative to Fechnerian models.

The class of simply scalable binary random choice models on a finite set $X$ was axiomatically characterized by Tversky and Russo (1969) by means of SST* and the -almost always assumed- Positivity axiom that requires all choice probabilities to be strictly positive. Fechnerian models on the other hand were recently axiomatized by Fudenberg et al. (2015) by means of Positivity and an Acyclicity axiom. An implication of our analysis is that $\rho$ is simply scalable if and only if the $\rho$-induced random preference intensity relation $\succsim$ is representable by a preference intensity function, and that $\rho$ is Fechnerian if and only if this $\succsim$ admits an additive such representation.

\section{Revealed preference intensity}

Behavioural datasets arising from two-alternative forced-choice experiments are very common in economics, psychology and neuroscience. In many of those experiments an additional variable with intensity-revealing potential is also elicited alongside the decision maker's choice at each menu. Examples of such additional observables were mentioned in Section 2.

Focusing on the case where the additional observable with intensity-revealing potential is response times, the first study that analyzed such extended data in the deterministic revealed-preference tradition was Echenique and Saito (2017). For the testable case of finite data, these authors proposed a notion of rationalizability that builds on the pseudo-cardinal utility-difference model in (3) and identified a testable axiom on such data that is necessary and sufficient for them to be utility-difference rationalizable. ${ }^{8}$ This notion requires the existence of a utility function $u: X \rightarrow \mathbb{R}$ and a strictly decreasing function ${ }^{9} f: \mathbb{R} \rightarrow \mathbb{R}$ such that

$$
\begin{array}{ccc}
C(\{a, b\})=\{a\} & \Longrightarrow & u(a)>u(b), \\
u(a)-u(b) & = & f(t(a, b)),
\end{array}
$$

\footnotetext{
${ }^{8}$ This model was labelled "response-time representation" by the authors. We use the term "utility-difference rationalization" instead as it is more general and also in line with the broader view that we adopt in this paper about potential sources of intensity-revealing data.

${ }^{9} \mathrm{~A}$ strictly decreasing $f$ allows the model to capture the intuition that response times are lower when the intensity difference between the two alternatives is higher, and is in line with predictions made by the "drift-diffusion model" (see, for example, Ratcliff and McKoon, 2008; Konovalov and Krajbich, 2019; Baldassi et al., 2020). The case where $f$ is strictly increasing is also covered in Echenique and Saito (2017).
} 
where $C(\{a, b\})=\{a\}$ means that $a$ is chosen over $b$ at menu $\{a, b\}$ and $t(a, b)$ is the observed response time for this choice of $a$ over $b$. The authors' Strong Compensation axiom that characterizes the model in effect provides the first non-parametric theoretical revealed preference intensity test for this class of behavioural data.

Despite the novelty of this analysis, however, the existence of a utility-difference representation that lies at the heart of such a utility-difference rationalization rules out some classes of perfectly consistent behaviour (cf Example 1). Moreover, as the authors highlighted, their Strong Compensation axiom does generate interpretable restrictions such as (Decreasing) Monotonicity $[t(a, c)<t(a, b), t(b, c)]$ and Time Transitivity $\left[t(a, b) \leq t\left(a^{\prime}, b^{\prime}\right)\right.$ and $t(b, c) \leq$ $t\left(b^{\prime}, c^{\prime}\right)$ implies $t(a, c) \leq t\left(a^{\prime}, c^{\prime}\right)$, which in turn can be thought of as the response-time analogs of Strong Stochastic Transitivity and Debreu's (1958) Stochastic Monotonicity axiom on binary stochastic choices, respectively. Yet, similar to the Cancellation axiom (Scott, 1964) that characterizes utility-difference representations, there are many additional restrictions imposed by Strong Compensation that are elusive because there seems to be no simple way to summarize what the axiom says about behaviour.

Informed by the above, we will now employ the preference intensity model that is laid out in (4a)-(4c) and follow a purely constructive approach to generalize the analysis in Echenique and Saito (2017) by relaxing the restrictions imposed on the data through a simple Congruence-like axiom (Richter, 1966). In addition, and although this change is primarily notational and intepretational rather than formal, to facilitate applications of these non-parametric tests in experimental and empirical economics we introduce a more general and unifying definition of a behavioural dataset that allows for the additional non-choice observable to be any relevant menu-specific variable with intensity-revealing potential such as those mentioned above, with response times being a special case.

Definition 5. A binary behavioural dataset $\mathcal{D}=\left\{\left\{a_{i}, b_{i}\right\}, C\left(\left\{a_{i}, b_{i}\right\}\right), r_{\left\{a_{i}, b_{i}\right\}}\right\}_{i=1}^{k}$ on a finite set $X$ is a collection of triples consisting of a binary menu $\left\{a_{i}, b_{i}\right\}$, the observed choice $C\left(\left\{a_{i}, b_{i}\right\}\right)$ at that menu, and the value $r_{\left\{a_{i}, b_{i}\right\}}$ of an observable and menu-specific intensity-revealing variable/resource.

From now on, we will use the notation $a>^{B} b$ whenever $C(\{a, b\})=\{a\}$ for some menu $\{a, b\}$ in $\mathcal{D}$, where $>^{B}$ denotes the (binary) revealed preference relation.

Definition 6. A binary behavioural dataset $\mathcal{D}$ on a finite set $X$ is preference-intensity rationalizable if there exist functions $s: X \times X \rightarrow \mathbb{R}$ and $f: \mathbb{R} \rightarrow \mathbb{R}$, with $f$ strictly monotonic, such that, for all $a, b, c \in X$,

$$
\begin{array}{rcl}
a>^{B} b & \Longrightarrow & s(a, b)>0 \\
s(a, b) & =f\left(r_{\{a, b\}}\right)= & -s(b, a) \\
s(a, b)>0, s(b, c)>0 & \Longrightarrow & s(a, c) \geq \max \{s(a, b), s(b, c)\} .
\end{array}
$$

In words, $\mathcal{D}$ is rationalizable in the above sense if: (i) a preference intensity function $s$ can be constructed so that each pair of alternatives $(a, b)$ where $a$ is revealed preferred to $b$ is associated with a strictly positive value; (ii) this value is itself a strictly monotonic function of the intensity-revealing resource at $\{a, b\}$; (iii) $s$ also satisfies a general acyclic version of the lateral consistency requirement that still ensures conceptual harmony between and within revealed preferences and intensities.

Turning to the model's empirical content, the following axiom -laid out in two versions, with the appropriate one for the given task being left to the analyst to determine- combines Richter's (1966) Congruence axiom in the present binary-choice setting with a weak and intuitive monotonicity requirement.

\section{Congruent Monotonicity - Positive}

If $a_{1}>^{B} a_{2} \cdots>^{B} a_{n}$ and $1<i<n$, then $a_{n} \nsucc^{B} a_{1}, r_{\left\{a_{1}, a_{i}\right\}} \ngtr r_{\left\{a_{1}, a_{n}\right\}}$ and $r_{\left\{a_{i}, a_{n}\right\}} \ngtr r_{\left\{a_{1}, a_{n}\right\}}$.

\section{Congruent Monotonicity - Negative}

If $a_{1}>^{B} a_{2} \cdots>^{B} a_{n}$ and $1<i<n$, then $a_{n} \nsucc^{B} a_{1}, r_{\left\{a_{1}, a_{i}\right\}} \nless r_{\left\{a_{1}, a_{n}\right\}}$ and $r_{\left\{a_{i}, a_{n}\right\}} \nless r_{\left\{a_{1}, a_{n}\right\}}$.

The novel monotonicity part of the axiom requires the value of the intensity-revealing variable to be weakly monotonic for every sequence of alternatives where any two consecutive elements in the sequence are related by revealed preference. Specifically, for an arbitrary such sequence $a_{1}, \ldots, a_{n}$, if $a_{1}>^{B} a_{i}$ and $i<i<n$, then with $a_{1}$ and $a_{n}$ being further apart from each other in the decision maker's indirect revealed preference relation than $a_{1}$ and $a_{i}$ are, the intensity that 
is revealed between $a_{1}$ and $a_{i}$ can be no higher than that revealed between $a_{1}$ and $a_{n}$ if the latter also happens to be observed. But since this may not actually be observed, the condition -in the positive variant of the axiom- is written as $r_{\left\{a_{1}, a_{i}\right\}} \ngtr r_{\left\{a_{1}, a_{n}\right\}}$ rather than $r_{\left\{a_{1}, a_{i}\right\}} \leq r_{\left\{a_{1}, a_{n}\right\}}$. The intuition for requiring $r_{\left\{a_{i}, a_{n}\right\}} \ngtr r_{\left\{a_{1}, a_{n}\right\}}$ is analogous. Congruent Monotonicity therefore imposes a cross-modal consistency requirement whereby the agent's revealed preferences and intensities are intuitively aligned. A dataset $\mathcal{D}$ will be said to satisfy Congruent Monotonicity if it satisfies either of the above two versions of the axiom for all observations contained in it.

Theorem 3. The following are equivalent for a binary behavioural dataset $\mathcal{D}$ on a finite set $X$ :

1. D satisfies Congruent Monotonicity.

2. $\mathcal{D}$ is preference-intensity rationalizable.

By analogy with the logical link between preference intensity functions and (pseudo-)cardinal utility functions that was mentioned in Section 4 , we also observe that a dataset $\mathcal{D}$ is utility-difference rationalizable in the sense of Echenique and Saito (2017) if and only if it is rationalizable by an additive preference intensity function.

Example 3. Suppose that $X:=\{a, b, c, d\}$ and consider the dataset $\mathcal{D}$ below where, without loss of generality, lower values of $r_{\{.\}}$point to higher intensities (cf response times):

$$
\begin{gathered}
a>^{B} b, \quad b>^{B} c, \quad c>^{B} d, \quad a>^{B} c, \quad a>^{B} d, \quad b>^{B} d \\
r_{\{a, d\}}<r_{\{b, d\}}<r_{\{a, c\}}<r_{\{b, c\}}<r_{\{a, b\}}<r_{\{c, d\}} .
\end{gathered}
$$

Notice that the the $r_{\{\cdot\}}$-ordering is as in the intensity relation $\succsim$ of Example 1, which induces the preferences on $X$ that coincide with the revealed preferences $>^{B}$. It is immediate, therefore, that $\mathcal{D}$ satisfies Congruent Monotonicity and is preference-intensity rationalizable, e.g. by the pair $(s, f)$ such that $s\left(a^{\prime}, b^{\prime}\right) \equiv f\left(r_{\left\{a^{\prime}, b^{\prime}\right\}}\right):=\frac{1}{r_{\left\{a^{\prime}, b^{\prime}\right\}}}$ if $a^{\prime}>^{B} b^{\prime}$ and $s\left(a^{\prime}, b^{\prime}\right)=-s\left(b^{\prime}, a^{\prime}\right)$ if $b^{\prime}>^{B} a^{\prime}$. Finally, since the revealed intensity ordering that is defined by the $r_{\{\cdot\}}$ data is not utility-difference representable (cf Example 1), it does not admit a utility-difference rationalization either.

\section{Concluding remarks}

We analysed a general and tractable model of preference intensity functions that generalizes those that belong to the utility-difference class in intuitive ways. In the spirit of the revealed-preference analysis tradition, the empirically testable behavioural content of the model was also identified on behavioural datasets that include choices from binary menus together with additional menu-specific observables with intensity-revealing potential. The proposed model can be thought of as the simplest analog of ordinal utility functions that allows for a decision maker's preferences and preference intensity comparisons to be represented in a genuinely ordinal way, without introducing any deviations from conventional notions of rationality on either of these relations, and at the same time without assuming that preference intensity comparisons are as if they were made by precision instruments.

\section{Appendix A.}

\section{Proof of Theorem 1.}

We first establish the following auxiliary results.

Claim 1. If $\succsim$ satisfies Weak Order and Reversal, then $(a, b) \succsim(b, a)$ and $(c, d) \succsim(d, c)$ implies $(a, b) \succsim(d, c)$. Moreover, if either $(a, b) \dot{>}(b, a)$ or $(b, c) \dot{>}(c, b)$ is also true, then $(a, b) \dot{>}(d, c)$.

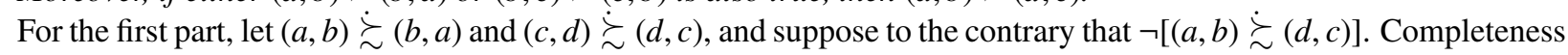
implies $(d, c) \dot{>}(a, b)$. Reversal further implies $(b, a) \dot{>}(c, d)$. Transitivity and

$$
(a, b) \succsim(b, a) \dot{>}(c, d) \succsim(d, c)
$$

now imply $(a, b) \dot{>}(d, c)$, a contradiction. For the second part, let $(a, b) \dot{>}(b, a),(c, d) \succsim(d, c)$ and suppose to the contrary that $(a, b) \dot{\sim}(d, c)$. Reversal implies $(c, d) \dot{\sim}(b, a)$. From $(a, b) \dot{>}(b, a)$, Transitivity and

$$
(d, c) \dot{\sim}(a, b) \dot{>}(b, a) \dot{\sim}(c, d)
$$


we get $(d, c) \dot{>}(c, d)$, which contradicts the postulate $(c, d) \succsim(d, c)$. The argument in the case where $(a, b) \succsim(b, a)$ and $(c, d) \dot{>}(d, c)$ is symmetric.

Claim 2. If $\succsim$ satisfies Weak Order, Reversal and Translocation Consistency, then $\succsim$ is a weak order on X.

Completeness of $\succsim$ immediately follows from its definition and the assumed Completeness of $\succsim$. For Transitivity, suppose $a \succsim b \succsim c$ and assume to the contrary that $c>a$. We have $(a, b) \succsim(b, a),(b, c) \succsim(c, b)$ and $(c, a) \dot{>}(a, c)$. In view of Claim $1,(a, b) \succsim(b, a)$ and $(c, a) \dot{>}(a, c)$ implies $(a, b) \dot{>}(a, c)$. By Reversal, $(c, a) \dot{>}(b, a)$. By Translocation Consistency, $(c, b) \dot{>}(b, c)$. This contradicts the postulate $(b, c) \succsim(c, b)$.

Claim 3. If $\dot{\succsim}$ satisfies Weak Order, Reversal and Translocation Consistency, then $(a, b) \succsim(b, a)$ and $(b, c) \succsim(c, b)$ implies $(a, c) \succsim(a, b)$ and $(a, c) \succsim(b, c)$.

Suppose $(a, b) \succsim(b, a)$ and $(b, c) \succsim(c, b)$ and assume to the contrary that $(a, b) \dot{\succ}(a, c)$. By Claim $1,(a, b) \succsim(b, a)$ and $(a, b) \dot{>}(a, c)$ implies $(a, b) \dot{>}(b, a)$. Similarly, $(b, c) \dot{\succsim}(c, b)$ and $(a, b) \dot{>}(a, c)$ implies $(b, c) \dot{>}(a, c)$. Moreover, $(a, b) \dot{>}(b, a)$ and $(b, c) \dot{>}(a, c)$ implies $(a, b) \dot{>}(a, c)$. By Reversal, $(c, a) \dot{>}(b, a)$. By Translocation Consistency, $(c, b) \dot{>}(b, c)$. This contradicts the postulate $(b, c) \succsim(c, b)$. The implication $(a, c) \succsim(b, c)$ is established symmetrically.

$1 \Rightarrow 2$. Suppose $(a, c) \succsim(b, c)$ and assume to the contrary that $\neg[(a, d) \succsim(b, d)]$. By Completeness, this implies $(b, d) \dot{>}(a, d)$. Translocation Consistency and $(a, c) \succsim(b, c)$ jointly imply $(a, b) \succsim(b, a)$. Translocation Consistency and $(b, d) \dot{>}(a, d)$ also imply $(b, a) \dot{>}(a, b)$, a contradiction.

$2 \Rightarrow 1$. Suppose $(a, c) \succsim(b, c)$. Together with Separability and Reversal, this implies $(a, b) \succsim(b, b) \succsim(b, a)$ and, by Transitivity, $(a, b) \grave{\succsim}(b, a)$.

$1 \Rightarrow 3$. Note first that, since $X$ is finite and $\succsim$ is a weak order on $X$ (Claim 2), there exist $k \sim$-equivalence classes [ $\left.a_{i}\right]$ which, with a slight abuse of notation, can be strictly ordered as

$$
\left[a_{1}\right]>\ldots>\left[a_{k}\right] .
$$

The above ordering will be held fixed throughout the proof. In particular, it is understood that, for any $i \leq k$, $a, b \in\left[a_{i}\right] \Leftrightarrow a \sim b$ and also that, for any $i<j, a \in\left[a_{i}\right]$ and $b \in\left[a_{j}\right] \Leftrightarrow a>b$.

Let the $\sim$-quotient set of $X$ be defined by $X_{\sim} \equiv \mathcal{X}:=\left\{\left[a_{1}\right], \ldots,\left[a_{k}\right]\right\}$. Let also

$$
A:=\left\{\left[a_{i}\right] \times\left[a_{j}\right] \in \mathcal{X} \times \mathcal{X}: i<j\right\}
$$

and

$$
Q_{>}\left(a_{i}, a_{j}\right):=\left\{\left[a_{h}\right] \times\left[a_{s}\right] \in A:\left(a_{i}, a_{j}\right) \dot{>}\left(a_{h}, a_{s}\right)\right\}
$$

That is, $\left[a_{h}\right] \times\left[a_{s}\right] \in Q_{\dot{ }}\left(a_{i}, a_{j}\right)$ if and only if, for all $\left(a_{h}, a_{s}\right) \in\left[a_{h}\right] \times\left[a_{s}\right], a_{h}>a_{s}$ and $\left(a_{i}, a_{j}\right)>\left(a_{h}, a_{s}\right)$. Notice that $Q_{\dot{ }}\left(a_{i}, a_{j}\right) \neq \emptyset$ implies $i<j$ but the converse is not true in general.

Now define the function $s: X \times X \rightarrow \mathbb{R}$ by

$$
s\left(a_{i}, a_{j}\right):= \begin{cases}1+\left|Q_{>}\left(a_{i}, a_{j}\right)\right|, & \text { if } i<j \\ 0, & \text { if } i=j \\ -s\left(a_{j}, a_{i}\right), & \text { if } i>j\end{cases}
$$

Note that this $s$ is well-defined in $X \times X$ since $\left(a_{i}, a_{j}\right) \in X \times X$ if and only if $\left(a_{i}, a_{j}\right) \in\left[a_{i}\right] \times\left[a_{j}\right]$ for some $\left[a_{i}\right],\left[a_{j}\right] \in \mathcal{X}$ where exactly one of $i<j, i=j$ and $i>j$ is true. Moreover, $s$ satisfies (4b) by construction. We will show that $s$ also satisfies (4a), and it will then follow from Claim 3 that $s$ obeys (4c) as well.

Notice first that it follows from the definitions of $s$ and $Q_{>}(\cdot)$, and also from $\left[a_{1}\right]>\ldots>\left[a_{k}\right]$, that $s\left(a_{i}, a_{j}\right)>$ $0 \Leftrightarrow a_{i}>a_{j}$ and $s\left(a_{i}, a_{j}\right)=0 \Leftrightarrow a_{i} \sim a_{j}$. Now suppose $\left(a_{j}, a_{l}\right) \succsim\left(a_{m}, a_{n}\right)$ and assume $j \leq l$. It holds that 
$\left[a_{m}\right] \times\left[a_{n}\right] \in Q_{\succ}\left(a_{j}, a_{l}\right)$ or $Q_{\succ}\left(a_{j}, a_{l}\right)=\emptyset$. Given the definitions of $s$ and $Q_{\succ}(\cdot)$, the first case implies $s\left(a_{j}, a_{l}\right)>$ $s\left(a_{m}, a_{n}\right)$ because $\left(a_{j}, a_{l}\right) \dot{>}\left(a_{m}, a_{n}\right)$ and therefore $Q_{\dot{ }}\left(a_{j}, a_{l}\right) \supset Q_{\dot{ }}\left(a_{m}, a_{n}\right)$ since $\dot{\succsim}$ is a weak order on $X \times X$. The second case, $Q_{\dot{ }}\left(a_{j}, a_{l}\right)=\emptyset$, implies $s\left(a_{j}, a_{l}\right)=1$. Moreover, if $Q_{\dot{ }}\left(a_{j}, a_{l}\right)=\emptyset$ and $m \leq n$, then $\left(a_{j}, a_{l}\right) \succsim\left(a_{m}, a_{n}\right)$ implies $\left(a_{j}, a_{l}\right) \dot{\sim}\left(a_{m}, a_{n}\right)$, which further implies $s\left(a_{m}, a_{n}\right)=1=s\left(a_{j}, a_{l}\right)$. On the other hand, $Q_{>}\left(a_{j}, a_{l}\right)=\emptyset$ and $m>n$ implies $s\left(a_{m}, a_{n}\right)<0<s\left(a_{j}, a_{l}\right)=1$. Assume now that $j>l$. In view of Claim 1 , this implies $m>n$. Reversal now implies $\left(a_{n}, a_{m}\right) \succsim\left(a_{l}, a_{j}\right)$. Applying the above argument to this case establishes that $s\left(a_{n}, a_{m}\right) \geq s\left(a_{l}, a_{j}\right)$ and, given that $s(a, b)=-s(b, a)$ for all $a, b \in X$ by construction, $s\left(a_{j}, a_{l}\right) \geq s\left(a_{m}, a_{n}\right)$. Thus, for all $a_{j}, a_{l}, a_{m}, a_{n} \in X$, $\left(a_{j}, a_{l}\right) \succsim\left(a_{m}, a_{n}\right)$ implies $s\left(a_{j}, a_{l}\right) \geq s\left(a_{m}, a_{n}\right)$. Conversely, suppose $s\left(a_{j}, a_{l}\right) \geq s\left(a_{m}, a_{n}\right)$. Assume to the contrary that

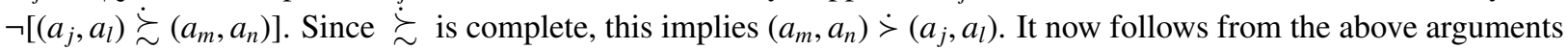

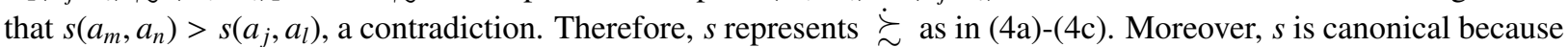
$s(X \times X)$ is a set of consecutive integers that is symmetric around 0 , by construction.

To establish the uniqueness property, let $s$ be a preference intensity function that represents $\succsim$ and let $t$ be an odd and strictly increasing transformation of $s$. Since $s(a, b)=-s(b, a)$ and $t(a, b)=f(s(a, b))$ for some function $f: \mathbb{R} \rightarrow \mathbb{R}$ that is odd in $s(X \times X)$, we have

$$
t(a, b)=f(s(a, b))=-f(-s(a, b))=-f(s(b, a))=-t(b, a) .
$$

Now suppose $(a, b) \succsim(c, d)$. This is equivalent to $s(a, b) \geq s(c, d)$. Since $t$ is a strictly increasing transformation of $s$, it follows that $t(a, b) \geq t(c, d)$ too. Conversely, suppose $\dot{\succsim}$ is represented by two distinct preference intensity functions $s$ and $t$. Let $t:=f \circ s$ for some function $f: \mathbb{R} \rightarrow \mathbb{R}$. Suppose $f(-z) \neq-f(z)$ for some $z \in s(X \times X)$. Let $z=s(a, b)$. Since $s(a, b)=-s(b, a)$ and $t(a, b)=f(s(a, b))$ by assumption, it follows that $t(b, a)=f(s(b, a))=f(-s(a, b)) \neq-f(s(a, b))=-t(a, b)$, which contradicts the assumption that $t$ represents $\succsim$. Therefore, $f$ is odd in $s(X \times X)$. Now suppose $f(z) \leq f\left(z^{\prime}\right)$ for some $z, z^{\prime} \in s(X \times X)$ such that $z>z^{\prime}$. Suppose $z=s(a, b)$ and $z^{\prime}=s(c, d)$. By assumption, $(a, b) \dot{>}(c, d)$. Since $f(z)=f(s(a, b))=t(a, b) \leq t(c, d)=f(s(c, d))=f\left(z^{\prime}\right)$, this again contradicts the assumption that $t$ represents $\succsim$. Therefore, $f$ is strictly increasing in $s(X \times X)$.

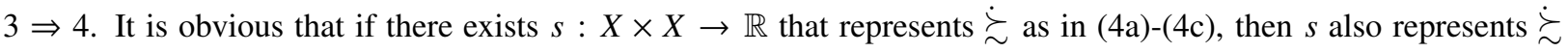
as in (5a)-(5c). The relevant part in the proof that $3 \Rightarrow 4$ can be invoked to also establish uniqueness up to a strictly increasing transformation of an arbitrary $g: X \times X \rightarrow \mathbb{R}$ that represents $\dot{\succsim}$ as in (5a)-(5c).

$4 \Rightarrow 1$. Weak Order is implied by (5a) and Reversal is implied by (5b). To show that Translocation Consistency is also implied by (5a)-(5c), suppose to the contrary that $(a, c) \succsim(b, c)$ and $(b, a) \dot{>}(a, b)$. We have $g(a, c) \geq g(b, c)$ and $g(b, a)>g(a, b)$. Notice that, by (5a) and (5b), $g(b, a)>g(a, b)$ implies $g(b, a)>g(a, a)>g(a, b)$.

Suppose first that $g(b, c) \geq g(b, a)$. We then have $g(a, c) \geq g(b, c) \geq g(b, a)>g(a, a)>g(a, b)$. Hence, $g(a, c)>$ $g(a, a)>g(a, b)$ and, by (5b), $g(b, a)>g(a, a)>g(c, a)$. It follows then that $g(b, a)>g(a, a), g(a, c)>g(a, a)$ and, by $(5 c), g(b, c)>g(a, c)$. This is a contradiction.

Now suppose $g(b, a)>g(b, c)$ instead. Then, either $g(b, a)>g(a, c)$ or $g(a, c) \geq g(b, a)$ is also true. Consider the former case first. We have $g(b, a)>g(a, a)>g(a, b)$ and $g(b, a)>g(a, c) \geq g(b, c)$. Suppose $g(a, c) \geq g(a, a)$. Then, by (5c), $g(b, a)>g(a, a)$ and $g(a, c) \geq g(a, a)$ implies $g(b, c) \geq g(b, a)$, which contradicts the first postulate of this paragraph. Now suppose $g(a, a)>g(a, c)$ instead. We have $g(a, a)>g(a, c) \geq g(b, c)$. By (5b), this implies $g(c, b)>g(a, a)$. By (5c), moreover, this and $g(b, a)>g(a, a)$ together imply $g(c, a)>g(c, b)$. By (5b) again, this is equivalent to $g(b, c)>g(a, c)$, a contradiction. Consider, finally, the case where $g(a, c) \geq g(b, a)$. We have $g(a, c) \geq g(b, a)>g(a, a)>g(a, b)$ and $g(b, a)>g(b, c)$. Therefore, by $(5 c), g(b, a)>g(a, a)$ and $g(a, c)>g(a, a)$ implies $g(b, c)>g(b, a)$, also a contradiction.

\section{Proof of Theorem 2.}

$1 \Rightarrow 2$. Since $X^{2} \times X^{2}$ is separable and connected, it follows from the Eilenberg-Debreu Theorem (Eilenberg, 1941; Debreu, 1954; Bridges and Mehta, 1995) that Weak Order and Continuity together imply the existence of a continuous $s: X \times X \rightarrow \mathbb{R}$ such that $(a, b) \succsim(c, d) \Leftrightarrow s(a, b) \geq s(c, d)$. This fact and Reversal further imply that $s(d, c) \geq s(b, a)$ whenever $s(a, b) \geq s(c, d)$. Finally, given the above and Translocation Consistency, it follows from Claim 3 in the proof of Theorem 1 that $s(a, b) \geq s(d, d)$ and $s(b, c) \geq s(d, d)$ implies $s(a, c) \geq \max \{s(a, b), s(b, c)\}$. 
$2 \Rightarrow 1$. Continuity of $\succsim$ is directly implied by continuity of $s$. The remaining argument is as in the last part of the proof of Theorem 1 .

\section{Proof of Theorem 3.}

With a slight abuse of notation, we write $\{a, b\} \in \mathcal{D}$ and $r_{\{a, b\}} \in \mathcal{D}$ when it is understood that the choice from menu $\{a, b\}$ and the intensity observable $r_{\{a, b\}}$ at that menu are in $\mathcal{D}$. Moreover, without loss of generality, the proof assumes that Congruent Monotonicity - Negative is satisfied. The case where Congruent Monotonicity - Positive is satisfied instead can be dealt with in a symmetric way.

The argument for the $2 \Rightarrow 1$ part is easy and omitted. Now, for $a, b \in X$ such that $a \nsucc^{B} b$ and $b \nsucc^{B} a$-to be written $a \|^{B} b$ - we denote by $[a, b]$ the possibly empty collection of all maximal sequences $\left\{a_{1}, \ldots, a_{k}\right\}$ such that $a=a_{1}$, $b=a_{k}$ and either $a_{i}>^{B} a_{i+1}$ for all $i=1, \ldots, k-1$ or $a_{i+1}>^{B} a_{i}$ for all $i=1, \ldots, k-1$. The latter two situations will be denoted by $a>^{\widehat{B}} b$ and $b>^{\widehat{B}} a$, respectively, where $>^{\widehat{B}}$ is the indirect binary revealed preference relation. Moreover, if $a>^{B} b$ or $b>^{B} a$ is true, we write $[a, b]:=\{a, b\}$. Finally, if $a \nsucc^{\widehat{B}} b$ and $b \chi^{\widehat{B}} a$, we write $a \|^{\widehat{B}} b$. In light of these definitions, we have

$$
\begin{array}{ll}
{[a, b]=\{a, b\},} & \text { if } a>^{B} b \text { or } b>^{B} a \\
{[a, b] \ni\left\{a_{i}\right\}_{i=1}^{k \geq 3} \supset\{a, b\},} & \text { if } a \nsucc^{B} b, b \nsucc^{B} a \text { and } a>^{\widehat{B}} b \text { or } b>^{\widehat{B}} a \\
{[a, b]=\emptyset,} & \text { if } a \|^{\widehat{B}} b
\end{array}
$$

Now observe that, by the first requirement of Congruent Monotonicity, $a>^{\widehat{B}} b$ implies $b \nsucc^{\widehat{B}} a$. Hence, we have the following:

Observation. For all $a, b \in X$, exactly one of the following is true: $a>^{\widehat{B}} b ; b>^{\widehat{B}} a ; a \|^{\widehat{B}} b$.

Next, define the function $s: X \times X \rightarrow \mathbb{R}$ by

$$
s(a, b):= \begin{cases}\left(\min _{\left\{a_{i}\right\}_{i=1}^{k} \in[a, b]} \min _{\left\{a_{i}, a_{i+l}\right\} \in \mathcal{D},} r_{\left\{a_{i}, a_{i+l} \in[a, b]\right.} r_{\left.a_{i+l}\right\}}-\epsilon,\right. & \text { if } a>^{\widehat{B}} b \\ 0, & \text { if } a \|^{\widehat{B}} b \\ \epsilon-\left(\min _{\left\{a_{i}\right\}_{i=1}^{k} \in[a, b]} \min _{\left\{a_{i}, a_{i+l}\right\} \in \mathcal{D},} r_{a_{i}, a_{i+l} \in[a, b]} r_{\left\{a_{i}, a_{i+l}\right\}}\right)^{-1}, & \text { if } b>^{\widehat{B}} a\end{cases}
$$

where

$$
\epsilon:=\frac{1}{2} \min _{\{a, b\} \in \mathcal{D}} r_{\{a, b\}}
$$

In view of the Observation and the preceding remarks, $s$ is well-defined on $X \times X$.

Note next that for any function $f: \mathbb{R} \rightarrow \mathbb{R}$ with the property that

$$
f\left(r_{\{a, b\}}\right):= \begin{cases}\frac{1}{r_{\{a, b\}}}-\epsilon, & \text { if } a>^{B} b \\ \epsilon-\frac{1}{r_{\{a, b\}}}, & \text { if } b>^{B} a\end{cases}
$$

for all $r_{\{a, b\}} \in \mathcal{D}$, it holds that $s(a, b)=f\left(r_{\{a, b\}}\right)$ and $f$ is strictly decreasing in $r_{\{a, b\}}$. Moreover, $s(a, b)=-s(b, a)$ and $s(a, b)>0 \Leftrightarrow a>^{\widehat{B}} b$ also hold by construction (for the latter claim, recall also the definition of $\epsilon$ ). The above implies, in particular, that $a>^{B} b \Rightarrow s(a, b)>0$. Therefore, (11a) and (11b) hold. It remains to be verified that $s(a, b)>0$ and $s(b, c)>0$ implies $s(a, c) \geq \max \{s(a, b), s(b, c)\}$. 
To this end, note first that $\min \{s(a, b), s(b, c)\}>0$ implies $a>^{\widehat{B}} b$ and $b>^{\widehat{B}} c$, which in turn implies $a>^{\widehat{B}} c$. Therefore, $s(a, c) \geq 0$. The claim that $s(a, c) \geq \max \{s(a, b), s(b, c)\}$ will be established by showing that the second and third implications of Congruent Monotonicity necessitate

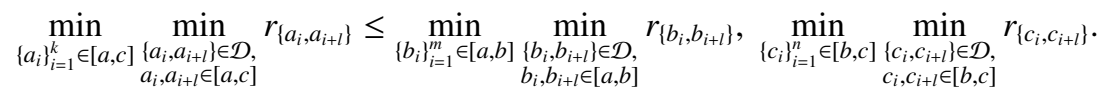

It is immediate that the minimum operators in (A.2) are redundant if $[a, b]=\{a, b\},[b, c]=\{b, c\}$ and $[a, c]=\{a, c\}$, i.e. when $a>^{B} b>^{B} c$ and $a>^{B} c$ are true. Similarly, the claim that (A.2) holds is also immediate if $[a, b] \neq\{a, b\}$, $[b, c] \neq\{b, c\}$ and there are unique maximal sequences $\left\{b_{i}\right\}_{i=1}^{m}$ and $\left\{c_{i}\right\}_{i=1}^{n}$ such that $[a, b]=\left\{b_{i}\right\}_{i=1}^{m},[b, c]=\left\{c_{i}\right\}_{i=1}^{n}$, in which case $[a, c]=\left\{b_{i}\right\}_{i=1}^{m} \cup\left\{c_{i}\right\}_{i=1}^{n}$ is also true. Now suppose $[a, b]$ or $[b, c]$ comprises more than one maximal sequence with the above properties. Then, each $\left\{a_{i}\right\}_{i=1}^{k} \in[a, c]$ can be written as

$$
\left\{a_{i}\right\}_{i=1}^{k}=\left\{b_{i}\right\}_{i=1}^{m} \cup\left\{c_{i}\right\}_{i=1}^{n} \quad \text { for some }\left\{b_{i}\right\}_{i=1}^{m} \in[a, b],\left\{c_{i}\right\}_{i=1}^{n} \in[b, c] .
$$

Suppose to the contrary that

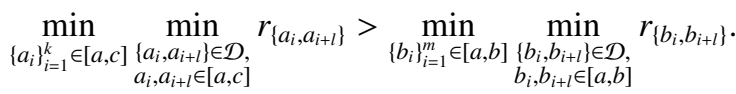

It holds that $\min _{\left\{b_{i}\right\}_{i=1}^{m} \in[a, b]} \min _{\substack{\left\{b_{i}, b_{i+1}\right\} \in \mathcal{D}, b_{i}, b_{i+1} \in[a, b]}} r_{\left\{b_{i}, b_{i+1}\right\}}=r_{\left\{a^{\prime}, b^{\prime}\right\}}$ for some $a^{\prime}, b^{\prime} \in X$ such that $a^{\prime}>^{B} b^{\prime}$. By (A.3) and Congruent Monotonicity,

$$
r_{\left\{a^{\prime}, b^{\prime}\right\}} \geq \min _{\left\{a_{i}\right\}_{i=1}^{k} \in[a, c]} \min _{\substack{\left\{a_{i}, a_{i+1}\right\} \in \mathcal{D}, a_{i}, a_{i+l} \in[a, c]}} r_{\left\{a_{i}, a_{i+l}\right\}}
$$

This contradicts (A.4). The case where $[a, b]$ is replaced by $[b, c]$ on the right hand side of (A.4) is similarly ruled out. Therefore, (A.2) holds.

\section{References}

[1] Abdellaoui, M., Barrios, C., Wakker, P.P.. Reconciling introspective utility with revealed preference: Experimental arguments based on prospect theory. Journal of Econometrics 2007;138:356-378.

[2] Aczél, J.. Lectures on Functional Equations and their Applications. New York: Academic Press, 1966.

[3] Adams, E.W.. Elements of a theory of inexact measurement. Philosophy of Science 1965;32:205-228.

[4] Alt, F.. Über die messbarkeit des nutzens. Zeitschrift für Nationalökonomie 1936;7:161-169.

[5] Alt, F.. On the measurability of utility. In: Chipman, J.S., Hurwicz, L., Richter, M.K., Sonnenschein, H.F., editors. Preferences, Utility and Demand. New York: Harcourt Brace Jovanovich; 1971. (English translation of Alt (1936) by S. Schach).

[6] Arrow, K.J.. Social Choice and Individual Values. New York: Wiley, 1951.

[7] Baccelli, J., Mongin, P.. Choice-based cardinal utility: A tribute to Patrick Suppes. Journal of Economic Methodology 2016;23:268-288.

[8] Baldassi, C., Cerreia-Vioglio, S., Maccheroni, F., Marinacci, M., Pirazzini, M.. A behavioral characterization of the drift diffusion model and its multi-alternative extension for choice under time pressure. Management Science 2020;66:5075-5093.

[9] Basu, K.. Determinateness of the utility function: Revisiting a controversy of the thirties. Review of Economic Studies 1982;49:307-311.

[10] Bossert, W., Weymark, J.A.. Utility in social choice. In: Barbera, S., Hammond, P., Seidl, C., editors. Handbook of Utility Theory, Volume 2: Extensions. Dordrecht: Kluwer; 2004. p. 1099-1177.

[11] Bridges, D.S., Mehta, G.B.. Representations of Preference Orderings. Berlin: Springer, 1995.

[12] Davidson, D., Marschak, J.. Experimental tests of a stochastic decision theory. In: Churchman, C.W., Ratoosh, P., editors. Measurement: Definitions and Theories. New York: Wiley; 1959. p. 233-269.

[13] Debreu, G.. Representation of a preference ordering by a numerical function. In: Thrall, R., Coombs, C., Davis, R., editors. Decision Processes. New York: Wiley; 1954. p. 159-165.

[14] Debreu, G.. Stochastic choice and cardinal utility. Econometrica 1958;26:440-444.

[15] Echenique, F., Saito, K.. Response time and utility. Journal of Economic Behavior \& Organization 2017;139:49-59.

[16] Eilenberg, S.. Ordered topological spaces. American Journal of Mathematics 1941;63:39-45.

[17] Ellingsen, T.. Cardinal utility: A history of hedonimetry. In: Allais, M., Hagen, O., editors. Cardinalism. Dordrecht: Kluwer; 1994. p. 105-165.

[18] Falmagne, J.C.. Elements of Psychophysical Theory. Oxford: Oxford University Press, 2002.

[19] Fleurbaey, M., Hammond, P.. Interpersonally comparable utility. In: Barbera, S., Hammond, P., Seidl, C., editors. Handbook of Utility Theory, Volume 2: Extensions. Dordrecht: Kluwer; 2004. p. 1179-1285.

[20] Frisch, R.. Sur un problème d'Économie pure. Norsk Matematisk Forenings Skrifter 1926;I-16:1-40. 
[21] Frisch, R.. On a problem in pure economics. In: Chipman, J.S., Hurwicz, L., Richter, M.K., Sonnenschein, H.F., editors. Preferences, Utility and Demand. New York: Harcourt Brace Jovanovich; 1971. (English translation of Frisch (1926) by J. S. Chipman).

[22] Fudenberg, D., Iijima, R., Strzalecki, T.. Stochastic choice and revealed perturbed utility. Econometrica 2015;83:2371-2409.

[23] Hammond, P.. Interpersonal comparisons of utility: Why and how they are and should be made. In: Elster, J., Roemer, J.E., editors. Interpersonal Comparisons of Well-Being. Cambridge: Cambridge University Press; 1991. p. 200-254.

[24] Köbberling, V.. Strength of preference and cardinal utility. Economic Theory 2006;27:375-391.

[25] Konovalov, A., Krajbich, I.. Revealed strength of preference: Inference from response times. Judgment and Decision Making 2019;14:381394.

[26] Krantz, D.H., Luce, R.D., Suppes, P., Tversky, A.. Foundations of Measurement, Volume I. New York: Wiley, 1971.

[27] Luce, R.D.. Semiorders and a theory of utility discrimination. Econometrica 1956;24:178-191.

[28] Luce, R.D., Krantz, D.H., Suppes, P., Tversky, A.. Foundations of Measurement, Volume III. San Diego and London: Academic Press, 1990.

[29] Luce, R.D., Raiffa, H.. Games and Decisions. New York: Wiley, 1957.

[30] Luce, R.D., Suppes, P.. Preference, utility and subjective probability. In: Luce, R.D., Bush, R.R., Galanter, E.H., editors. Handbook of Mathematical Psychology, Volume 3. New York: Wiley; 1965. p. 249-410.

[31] Mandler, M.. Dilemmas in Economic Theory. New York: Oxford University Press, 1999.

[32] Mandler, M.. Cardinality versus ordinality: A suggested compromise. American Economic Review 2006;96:1114-1136.

[33] von Neumann, J., Morgenstern, O.. Theory of Games and Economic Behavior. 2nd ed. Princeton: Princeton University Press, 1947.

[34] Phelps-Brown, E.H.. Notes on the determinateness of the utility function. Review of Economic Studies 1934;2:66-69.

[35] Ratcliff, R., McKoon, G.. The diffusion decision model: Theory and data for two-choice decision tasks. Neural Computation 2008;20:873922.

[36] Richter, M.K.. Revealed preference theory. Econometrica 1966;34:635-645.

[37] Roberts, F.S.. Measurement Theory with Applications to Decisionmaking, Utility and the Social Sciences. Reading, MA: Addison-Wesley, 1979.

[38] Samuelson, P.A.. The numerical representation of ordered classifications and the concept of utility. Review of Economic Studies 1938;6:6570.

[39] Schoemaker, P.J.H.. The expected utility model: Its variants, purposes, evidence and limitations. Journal of Economic Literature 1982;20:529-563.

[40] Scott, D.. Measurement structures and linear inequalities. Journal of Mathematical Psychology 1964;1:233-247.

[41] Shapley, L.S.. Cardinal utility from intensity comparisons. Technical Report; 1975.

[42] Suppes, P., Krantz, D.H., Luce, R.D., Tversky, A.. Foundations of Measurement, Volume II. San Diego and London: Academic Press, 1989.

[43] Suppes, P., Winet, M.. An axiomatization of utility based on the notion of utility differences. Management Science 1955;1:259-270.

[44] Tversky, A.. A general theory of polynomial conjoint measurement. Journal of Mathematical Psychology 1967;4:1-20.

[45] Tversky, A., Russo, J.E.. Similarity and substitutability in binary choices. Journal of Mathematical Psychology 1969;6:1-12. 